\title{
Efficacy and safety of elobixibat in hemodialysis patients with chronic constipation: a retrospective study
}

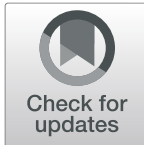

Takefumi Shono* and Hiroyuki Hyakutake

\begin{abstract}
Background: Hemodialysis patients are likely to develop constipation as a complication due to food and fluid restrictions. Elobixibat is a new laxative that inhibits the ileal bile acid transporter expressed in the terminal ileum, suppresses bile acid reabsorption, increases the amount of bile acid flowing into the colon lumen, and promotes water secretion in the colon and colon motility. In this study, we examined the efficacy and safety of elobixibat in maintenance hemodialysis patients with chronic constipation.
\end{abstract}

Methods: In a study conducted on maintenance hemodialysis patients with chronic constipation, comparisons were conducted retrospectively based on constipation symptoms such as the frequency of spontaneous bowel movements before elobixibat treatment and 12 weeks after the administration of elobixibat, as well as based on blood electrolyte levels.

Results: The study was conducted on 23 patients, 19 of whom were subjected to treatment efficacy analysis. The frequency of spontaneous bowel movements was $2.0 \pm 0.7$ times/week before administration of elobixibat and reached $4.9 \pm 2.3$ times/week after 12 weeks of administration. The increase started after 1 week of treatment. Elobixibat treatment improved the constipation score (baseline, $10.4 \pm 3.2$; after 12 weeks, $5.7 \pm 3.2$ ), the Bristol Stool Form Scale (baseline, $2.2 \pm 1.0$; after 12 weeks, $4.2 \pm 0.7$ ), and patient satisfaction regarding defecation (baseline, $2.9 \pm 0.9$; after 12 weeks, $1.7 \pm 1.1$ ). The interdialytic weight gain (IDWG) was $5.68 \pm 0.82 \%$ before initiation of treatment and decreased to $4.54 \pm 1.34 \%$ after 12 weeks. The serum inorganic phosphorus (IP) levels were $5.68 \pm$ $1.25 \mathrm{mg} / \mathrm{dL}$ and decreased to $4.93 \pm 1.10 \mathrm{mg} / \mathrm{dL}$ after 12 weeks.

Conclusions: Elobixibat was effective in improving constipation symptoms in maintenance hemodialysis patients with chronic constipation. Elobixibat also improved dialysis-related laboratory test results, such as a decrease in serum IP levels and IDWG.

Trial registration: UMIN Clinical Trials Registry, UMIN000037285, retrospectively registered

Keywords: Elobixibat, Hemodialysis, Constipation, Spontaneous bowel movements, Serum phosphorus levels, Interdialytic weight gain

\footnotetext{
* Correspondence: tkfm-shn.3@smile.ocn.ne.jp

Hyakutake Clinic, 2195-26, Ikari, Tagawa-Shi, Fukuoka 825-0001, Japan
}

C C The Author(s). 2020 Open Access This article is licensed under a Creative Commons Attribution 4.0 International License, which permits use, sharing, adaptation, distribution and reproduction in any medium or format, as long as you give appropriate credit to the original author(s) and the source, provide a link to the Creative Commons licence, and indicate if changes were made. The images or other third party material in this article are included in the article's Creative Commons licence, unless indicated otherwise in a credit line to the material. If material is not included in the article's Creative Commons licence and your intended use is not permitted by statutory regulation or exceeds the permitted use, you will need to obtain permission directly from the copyright holder. To view a copy of this licence, visit http://creativecommons.org/licenses/by/4.0/ The Creative Commons Public Domain Dedication waiver (http://creativecommons.org/publicdomain/zero/1.0/) applies to the data made available in this article, unless otherwise stated in a credit line to the data. 


\section{Background}

The total number of dialysis patients in Japan increases continually, and the proportion of elderly subjects in the dialysis population is also rising [1]. Hemodialysis patients are often complicated with constipation due to dietary and fluid restrictions, as well as due to the use of phosphate binders and potassium binders [2]. Constipation has been reported to cause a deterioration in the quality of life (QOL) of dialysis patients [3]. Given that constipation is associated with an increased risk of cardiovascular death $[4,5]$, treating constipation is important in dialysis patients who have a high risk of death from cardiovascular disease [6].

However, the treatment of chronic constipation in maintenance hemodialysis patients has several restrictions. Magnesium preparations are not recommended because of the increased risk of hypermagnesemia, and bulk-forming laxatives and invasive laxatives are not recommended because they require fluid intake. Therefore, medications for constipation treatment in hemodialysis patients must be carefully selected [2, 7-9]. New laxatives have recently been approved, including the epithelial function altering agents lubiprostone and linaclotide, the bile acid transporter inhibitor elobixibat, and the osmotic laxative polyethylene glycol; therefore, there are more options available for the treatment of constipation.

Elobixibat is a new laxative that inhibits the ileal bile acid transporter expressed in the epithelial cells of the terminal ileum; it inhibits bile acid reabsorption and increases the amount of bile acids flowing into the colon lumen [10]. Owing to the increased amount of bile acids in the colon, elobixibat increases water secretion into the large intestinal lumen and induces high amplitudepropagating pressure wave [11]. In other words, elobixibat has dual actions, which are water secretion into the large intestine and promotion of bowel movement. These reports suggest that elobixibat has a different mechanism of action from existing constipation treatments $[12,13]$. In a placebo-controlled, randomized, double-blind study and an open-label long-term administration study (52-week administration of elobixibat) conducted on Japanese patients with functional chronic constipation, elobixibat was effective for improving the frequency of spontaneous bowel movements. Its tolerability during long-term administration has also been confirmed [14]. However, its efficacy and safety in maintenance hemodialysis patients with chronic constipation have not yet been studied.

In our study aimed at examining the efficacy and safety of elobixibat in maintenance hemodialysis patients, the effect of treatment on constipation and the variations of blood electrolyte levels were confirmed.

\section{Methods}

Our retrospective observational study was conducted on maintenance hemodialysis patients who had received hemodialysis for 6 months or longer at Hyakutake Clinic between April 19, 2018, and January 18, 2019. The condition of the patients was consistent with the Rome IV criteria for the diagnosis of functional constipation [15]. The patients who had been taking oral elobixibat for 12 weeks or longer and whose effectiveness such as the frequency of spontaneous bowel movements was confirmed were evaluated. The following patients were excluded: patients suspected of having organic constipation, as well as patients who refused to allow their medical record information to be used for research purposes. This retrospective observational study was approved by the Yamauchi Clinic's Ethical Review Board (approval code 2019-06-00064) and was registered with the UMIN Clinical Trials Registry (UMIN-CTR, UMIN000037285).

Elobixibat therapy was started with a dose of $10 \mathrm{mg}$ once a day before meals and was adjusted between 5 and $15 \mathrm{mg}$ per day, depending on the symptoms. After initiation of oral elobixibat treatment, oral stimulant laxatives that had been taken by the patients before the study were continued unless the patients wanted otherwise, and non-stimulant laxatives (such as lubiprostone and magnesium oxide) were discontinued. Patients whose oral medications, other than laxatives and dialysis conditions, remained unchanged throughout the study were selected.

To evaluate the efficacy and safety of elobixibat, before initiation of treatment and at 1, 2, 3, 4, 6, 8, 10, and 12 weeks after initiation of treatment, the following were measured: the frequency of spontaneous bowel movements per week, Bristol Stool Form Scale (BSFS) [16], interdialytic weight gain (IDWG), and side effects. Before initiation of treatment and at 2, 4, 6, 8, 10, and 12 weeks after initiation of treatment, the following were measured: the serum levels of inorganic phosphorus (IP), potassium $(\mathrm{K})$, sodium $(\mathrm{Na})$, chlorine $(\mathrm{Cl})$, calcium $(\mathrm{Ca})$, and albumin (Alb) were measured. Before initiation of treatment and at 12 weeks after initiation of treatment, the following were measured: constipation score system (CSS) score [17], patient satisfaction regarding defecation (5 stages, namely from 0 "extremely satisfied" to 4 "extremely dissatisfied"), and serum levels of lowdensity lipoprotein cholesterol (LDL-C) were measured.

The results are presented as mean and standard deviation or median (minimum, maximum). Values measured before initiation of treatment and those measured after 12 weeks of treatment were compared using the paired $t$-test or the Wilcoxon signed-rank test, and $p<$ 0.05 was considered as the level of significance. The results of the classification of defecation according to the CSS score and the duration of disease as well as history of the present illness were not subjected to statistical analysis. In this study, statistical analysis was performed using the statistical calculation software R (Ver. 3.4.0 or later, R Foundation, Vienna, Austria). 
Table 1 Patient background

\begin{tabular}{ll}
\hline Number of patients (male/female) $(n)$ & $23(11 / 12)$ \\
\hline Age (years) & $72.2 \pm 8.5$ \\
Height (cm) & $155.61 \pm 11.35$ \\
Body weight (kg) & $52.01 \pm 12.05$ \\
Hemodialysis duration (years) & $6.0(1.0,35.0)$ \\
History of hemodialysis & \\
Diabetic nephropathy & 9 \\
Chronic glomerulonephritis & 8 \\
Nephrosclerosis & 2 \\
Polycystic kidney & 2 \\
Others & 2 \\
Complication & 23 \\
Hyperphosphatemia & 21 \\
Hypertension & 16 \\
Hyperlipidemia & 9 \\
Angina pectoris & 7 \\
Hypothyroidism & 3 \\
\hline
\end{tabular}

Category data are expressed as real values, and continuous values as mean \pm SD or median (min, max). Complications include duplication

\section{Results}

The characteristics of 23 study patients are shown in Table 1. There were 11 males and 12 females; the mean age was $72.2 \pm 8.5$ years. The hemodialysis period was $6.0(1.0,35.0)$ years. The present illness that resulted in the patient requiring hemodialysis was diabetic nephropathy in 9 patients, followed by chronic glomerulonephropathy in 8 . In terms of complications, 21 patients were complicated with hyperphosphatemia, including 16 who had been treated with phosphate binders.

There were 19 patients (82.6\%) who used other laxatives as pretreatment drugs. Among all patients, 4 patients reduced laxative use at the start of the elobixibat administration (reduced in 3 patients, discontinued in 1 patient), 9 patients changed laxatives, and 4 patients increased/added laxatives during the observation period. In these four cases, as-needed use of laxatives was allowed (including the glycerin enema). There was no change in laxatives for 6 patients (one of whom did not use a laxative as a pretreatment drug).

There were 16 patients (69.6\%) who used phosphorus binders as a pretreatment drug, and one patient was administered calcium carbonate. However, none of the patients added new phosphorus binders or changed their doses of phosphorus binders during the observation period. Thirteen patients were administered vitamin D. Three patients were administered calcimimetics drugs, one of whom increased the dose during the observation period.

Analysis of treatment efficacy was performed on 19 of the 23 patients $(2$ had stopped taking their medication, and the results of evaluation of efficacy could not be determined in 2). The frequency of spontaneous bowel movements showed a significant increase from $2.0 \pm 0.7$ times/ week before the initiation of treatment to $4.9 \pm 2.3$ times/ week after 12 weeks of treatment (Fig. 1). Further, the changes in the frequency of spontaneous bowel movements from the initiation of treatment until 12 weeks of treatment were as follows. The frequency of spontaneous bowel movements reached $3.1 \pm 1.6$ times/week after 1 week of treatment and $5.4 \pm 2.0$ times/week after 6 weeks

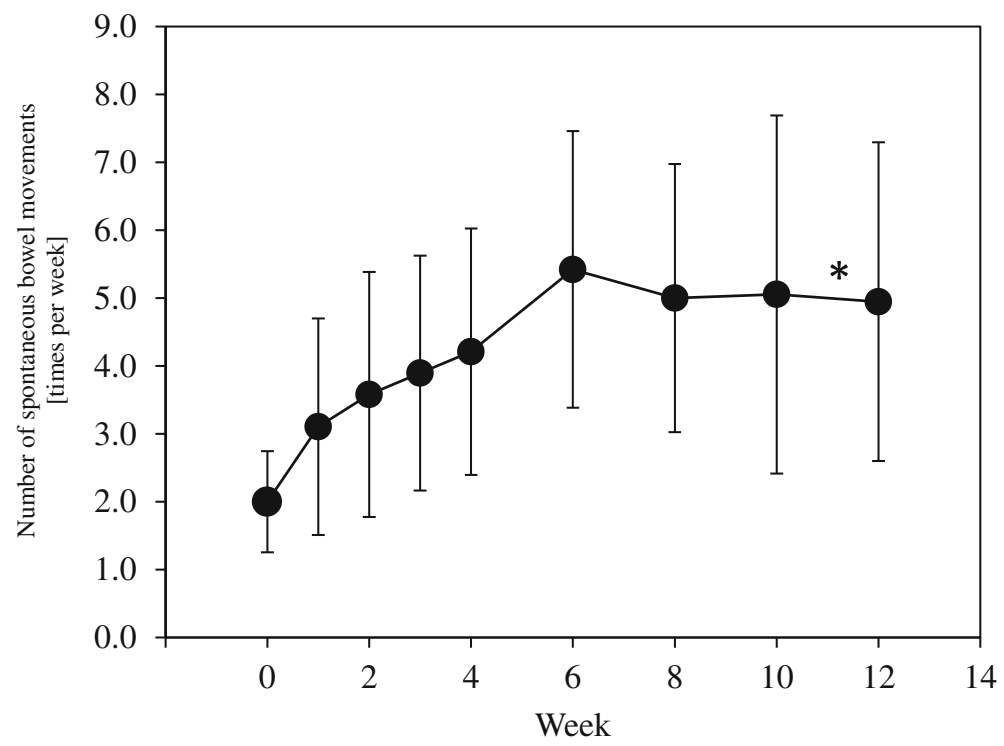

Fig. 1 Changes in the frequency of spontaneous bowel movements. ${ }^{*} p<0.05$ paired $t$-test (vs. before initiation of elobixibat treatment) 
Table 2 Comparison of constipation scores

\begin{tabular}{llll}
\hline Parameter & Mean \pm SD & & $p$ value \\
\cline { 2 - 3 } & Baseline & Week 12 after administration & $<0.001$ \\
\hline Total CSS score & $10.4 \pm 3.2$ & $5.7 \pm 3.2$ & 0.005 \\
Stool frequency & $0.9 \pm 0.7$ & $0.4 \pm 0.6$ & $<0.001$ \\
Dyschezia & $1.9 \pm 0.9$ & $1.3 \pm 0.8$ & 0.028 \\
A sensation of incomplete evacuation & $2.2 \pm 1.3$ & $0.5 \pm 1.0$ & 0.031 \\
Abdominal pain & $1.2 \pm 1.1$ & $0.6 \pm 0.8$ & 0.145 \\
Time to evacuation & $0.9 \pm 0.7$ & $0.8 \pm 0.4$ & 0.020 \\
Assisted bowel movements & $1.2 \pm 0.4$ & $0.3 \pm 0.5$ & $<0.001$ \\
Number of bowel movements failed/24h & $1.1 \pm 0.5$ & $1.3 \pm 1.1$ & - \\
Duration (years) of suffering from disturbed defecation & $1.1 \pm 0.9$ &
\end{tabular}

Statistical analysis was performed using the Wilcoxon signed-rank test

No statistical analysis was performed on the duration of suffering from disturbed defecation

of treatment, showing an increasing trend; later, it remained nearly constant up to 12 weeks of treatment (Fig. 1). The total CSS score improved significantly from $10.4 \pm 3.2$ before initiation of treatment to $5.7 \pm 3.2$ after 12 weeks of treatment. Significant improvements were found in terms of stool frequency, defecation difficulty, feeling of incomplete bowel emptying, abdominal pain, and the use or non-use of assistance for defecation, as well as the number of failed defecation attempts per $24 \mathrm{~h}$ (Table 2). The BSFS score, which was $2.2 \pm 1.0$ before treatment, also increased to $4.2 \pm 0.7$ after 12 weeks of treatment, showing that the stool shape had become nearly normal (the normal score is 4) (Fig. 2). Patient satisfaction regarding defecation was $2.9 \pm 0.9$ before initiation of treatment but reached $1.7 \pm 1.1$ after 12 weeks of treatment, showing a significant improvement (Fig. 3).
Before initiation of treatment, no patient selected "0: extremely satisfied" and " 1 : satisfied," but after 12 weeks of treatment, 3 (15.8\%) selected "0: extremely satisfied" and 4 (21.1\%) selected "1: satisfied," showing an increase in both categories.

Twelve weeks of treatment using elobixibat caused a significant decrease in the IDWG from $5.68 \pm 0.82 \%$ to $4.54 \pm$ $1.34 \%$ (Fig. 4). Furthermore, the IP level decreased significantly from $5.68 \pm 1.25 \mathrm{mg} / \mathrm{dL}$ before treatment to $4.93 \pm$ $1.10 \mathrm{mg} / \mathrm{dL}$ after 12 weeks of treatment (Fig. 5a). On the other hand, $\mathrm{K}, \mathrm{Na}, \mathrm{Cl}, \mathrm{Ca}$, Alb, and LDL-C levels showed no difference before treatment and after 12 weeks of treatment (K levels changed from $4.38 \pm 0.62$ to $4.15 \pm 0.42 \mathrm{mEq} / \mathrm{L}, \mathrm{Na}$ levels changed from $137.8 \pm 3.1$ to $137.5 \pm 3.5 \mathrm{mEq} / \mathrm{L}, \mathrm{Cl}$ levels changed from $102.1 \pm 4.0$ to $101.5 \pm 4.3 \mathrm{mEq} / \mathrm{L}, \mathrm{Ca}$ levels changed from $8.66 \pm 0.40$ to $8.68 \pm 0.46 \mathrm{mg} / \mathrm{dL}, \mathrm{Alb}$

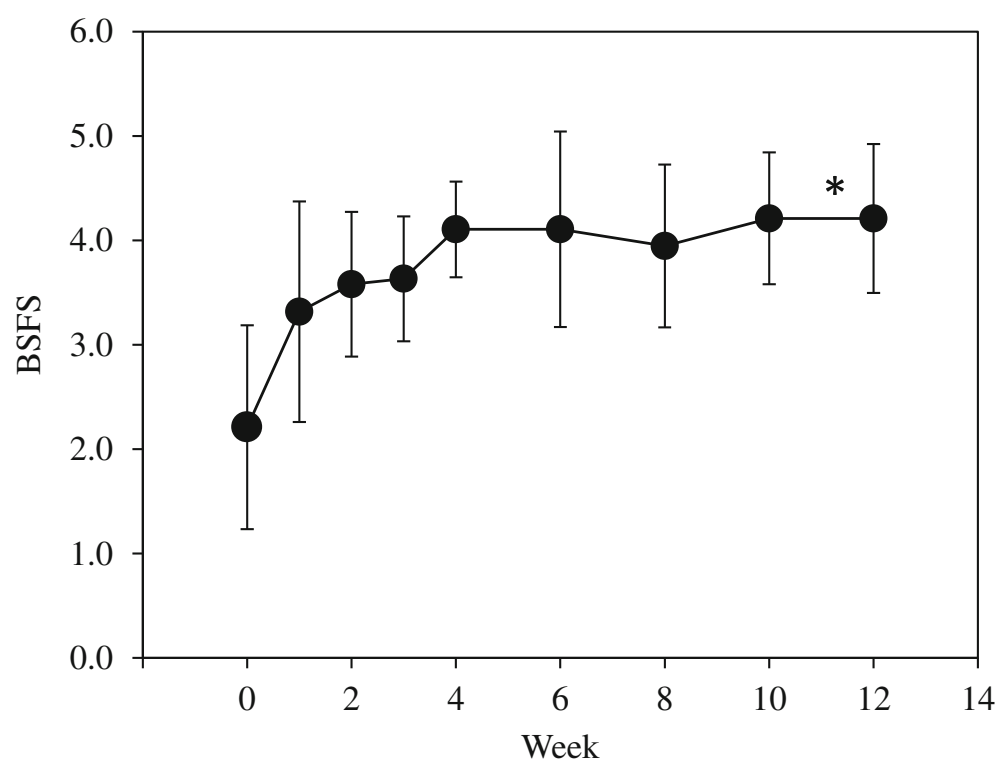

Fig. 2 Changes in the BSFS scores. ${ }^{*} p<0.05$ paired t-test (vs. before initiation of elobixibat treatment). BSFS, Bristol Stool Form Scale 


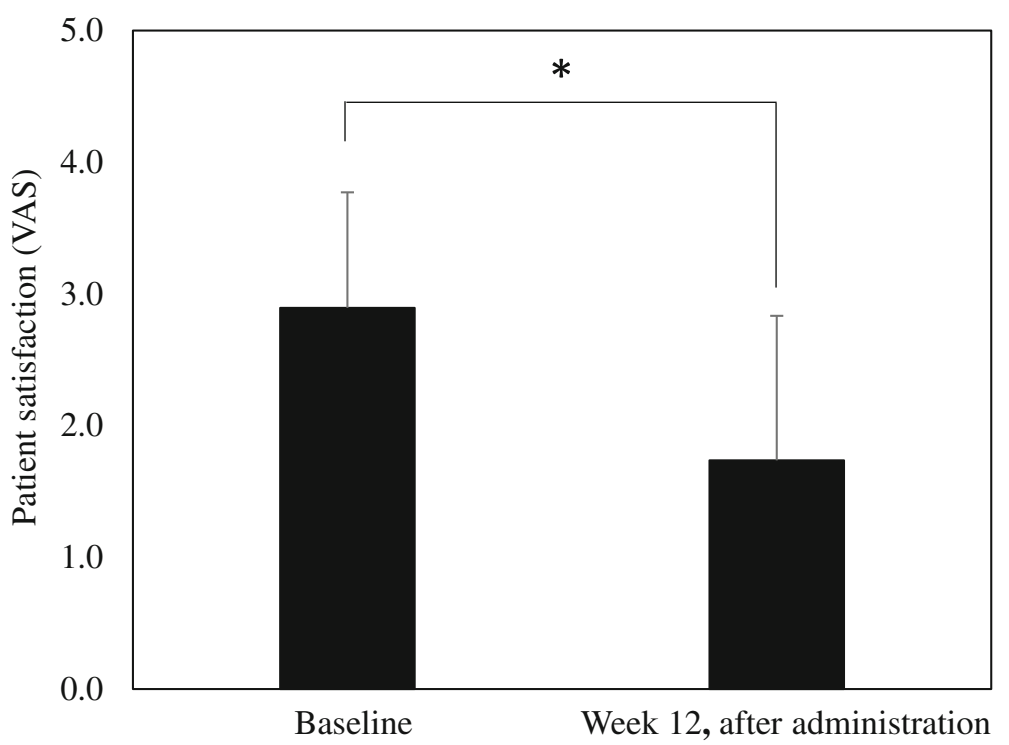

Fig. 3 Changes in VAS. ${ }^{*} p<0.05$ Wilcoxon signed-rank test. VAS, visual analog scale

levels changed from $3.44 \pm 0.30$ to $3.39 \pm 0.28 \mathrm{~g} / \mathrm{dL}$, and LDL-C levels changed from $80.2 \pm 32.0$ to $66.7 \pm 24.7 \mathrm{mg} /$ dL; Fig. 5b-f).

Stratified analyses were performed based on the presence or absence of diabetic nephropathy, the most common disorder that required hemodialysis (Table 3). In patients with diabetic nephropathy (8 cases), 12 weeks of treatment using elobixibat increased the frequency of spontaneous bowel movements from $1.8 \pm 0.5$ times/week to $4.9 \pm 2.4$ times/week and reduced the total CSS score from $10.6 \pm 3.6$ to $6.8 \pm 4.3$. Similarly, in patients with other disorders that required hemodialysis (11 cases), elobixibat treatment increased the frequency of spontaneous bowel movements from $2.2 \pm 0.9$ times/week to 5.0 \pm 2.4 times/week and reduced the total CSS score from $10.3 \pm 2.9$ to $5.0 \pm 2.1$. In all CSS subscores, decreased values were found after 12 weeks of oral treatment with elobixibat, with the exception of the duration of suffering from disturbed defecation and the use of assistance for defecation in patients with diabetic nephropathy.

Stratified analyses based on the use of phosphate binder were also performed (Table 4). In patients with phosphate binder treatment (14 cases), 12-week elobixibat treatment increased the frequency of spontaneous

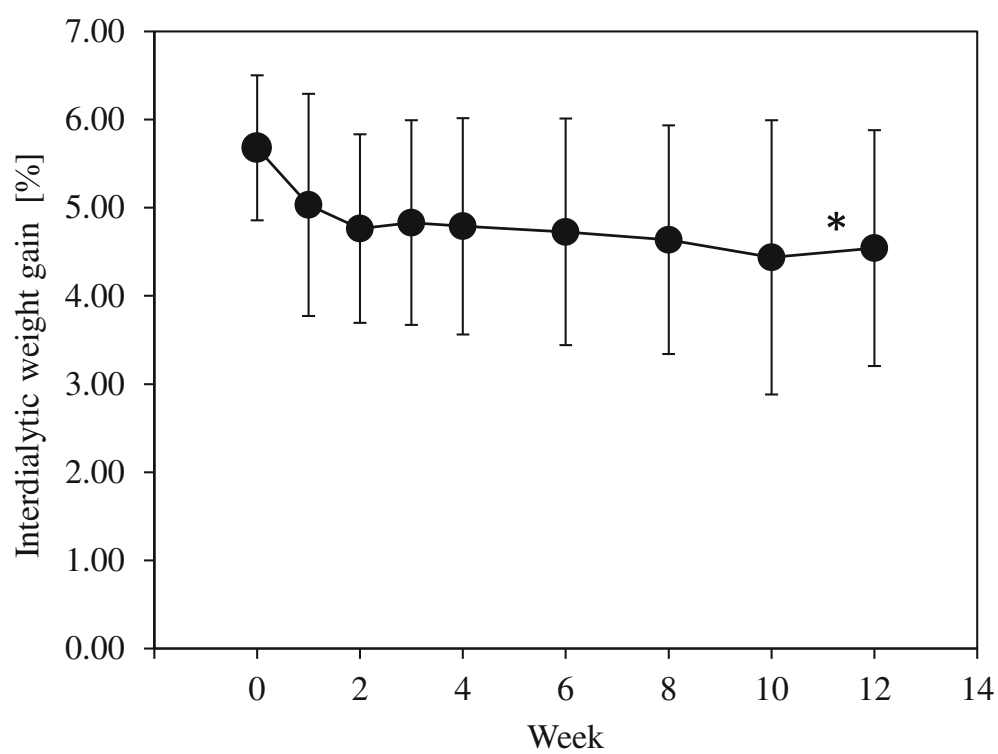

Fig. 4 Changes in IDWG. ${ }^{*} p<0.05$ paired $t$-test (vs. before initiation of elobixibat treatment). IDWG, interdialytic weight gain 

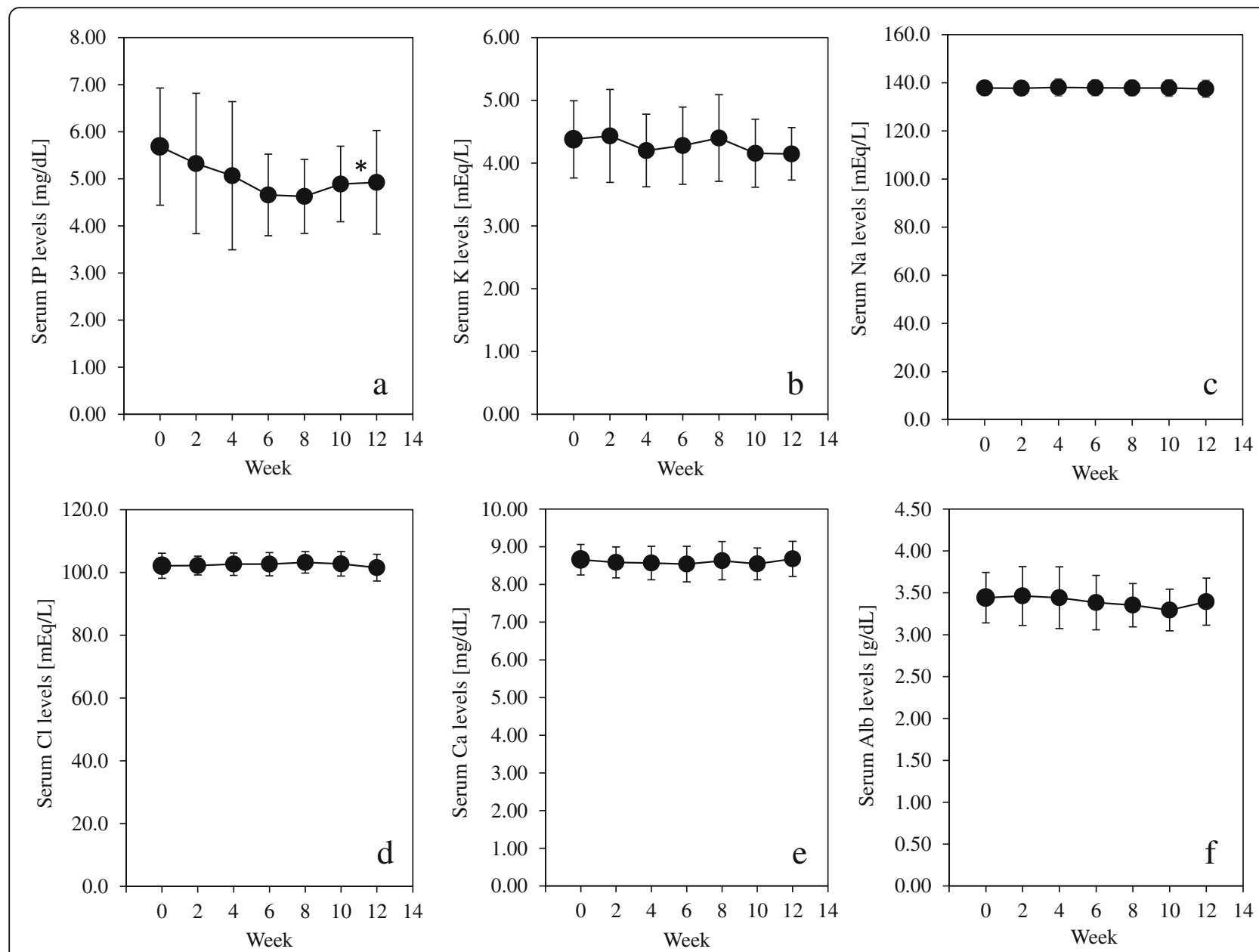

Fig. 5 Changes in serological test values. a Serum inorganic phosphorus (IP) levels. b Serum potassium (K) levels. c Serum sodium (Na) levels. d Serum chlorine (Cl) levels. e Serum calcium (Ca) concentrations. $\mathbf{f}$ Serum albumin (Alb) concentrations. ${ }^{*} p<0.05$ paired $t$-test (vs. before initiation of elobixibat treatment)

bowel movements from $1.8 \pm 0.6$ times/week to $4.9 \pm$ 2.3 times/week. Similarly, in the patients without phosphate binder treatment (5 cases), elobixibat treatment increased the frequency of spontaneous bowel movement from $2.6 \pm 0.9$ times/week to $5.2 \pm 2.6$ times/week. Regardless of the use of a phosphate binder, elobixibat treatment decreased the total CSS score and all CSS subscores, except for the duration of disturbed defecation.

Regarding safety, side effects were found in 5 $(21.7 \%)$ of the 23 patients: diarrhea was found in 5 (21.7\%), abdominal pain in $2(8.7 \%)$, and nausea in 1 $(4.3 \%)$. All side effects were mild, and none of the patients had to discontinue oral elobixibat treatment because of side effects. Dose reduction due to side effects was necessary in one patient who presented with diarrhea; no other patient was subjected to dosage change due to side effects. Further, all patients who developed side effects recovered within 1 week of onset.

\section{Discussion}

Constipation is highly frequent in maintenance hemodialysis patients because of comorbidities affecting bowel movement, such as diabetes mellitus, cerebrovascular disease, hypothyroidism, decreased physical activity, dietary restriction, fluid restriction, and the use of phosphate and potassium binders [2]. Our study showed that elobixibat increased the frequency of spontaneous bowel movements in maintenance hemodialysis patients with chronic constipation. Additionally, elobixibat also improved the total CSS score, the CSS scores (stool frequency, defecation difficulty, feeling of incomplete bowel emptying, the use or non-use of assistance for defecation, the number of failed defecation attempts per $24 \mathrm{~h}$ ), and the BSFS, as well as patient satisfaction regarding defecation. Those findings showed that in maintenance hemodialysis patients, elobixibat was also useful for the improvement of stool frequency, constipation symptoms, stool hardness, and patient satisfaction regarding defecation. 
Table 3 Comparison of spontaneous stool frequency and constipation score by clinical history

\begin{tabular}{|c|c|c|c|c|}
\hline \multirow[t]{2}{*}{ Parameter } & \multirow{2}{*}{$\begin{array}{l}\text { Current medical } \\
\text { history }\end{array}$} & \multicolumn{2}{|l|}{ Mean \pm SD } & \multirow[t]{2}{*}{$p$ value } \\
\hline & & Baseline & Week 12 after administration & \\
\hline \multirow[t]{2}{*}{ Spontaneous stool frequency } & Diabetic nephropathy & $1.8 \pm 0.5$ & $4.9 \pm 2.4$ & 0.009 \\
\hline & Others & $2.2 \pm 0.9$ & $5.0 \pm 2.4$ & $<0.001$ \\
\hline \multirow[t]{2}{*}{ Total CSS score } & Diabetic nephropathy & $10.6 \pm 3.6$ & $6.8 \pm 4.3$ & 0.014 \\
\hline & Others & $10.3 \pm 2.9$ & $5.0 \pm 2.1$ & 0.006 \\
\hline \multirow[t]{2}{*}{ Stool frequency } & Diabetic nephropathy & $1.1 \pm 0.4$ & $0.3 \pm 0.5$ & 0.011 \\
\hline & Others & $0.8 \pm 0.9$ & $0.5 \pm 0.7$ & 0.203 \\
\hline \multirow[t]{2}{*}{ Dyschezia } & Diabetic nephropathy & $1.5 \pm 0.8$ & $0.9 \pm 1.0$ & 0.120 \\
\hline & Others & $2.2 \pm 1.0$ & $0.4 \pm 0.7$ & 0.003 \\
\hline \multirow[t]{2}{*}{ A sensation of incomplete evacuation } & Diabetic nephropathy & $2.4 \pm 1.3$ & $1.6 \pm 1.6$ & 0.095 \\
\hline & Others & $2.0 \pm 1.3$ & $1.1 \pm 1.2$ & 0.138 \\
\hline \multirow[t]{2}{*}{ Abdominal pain } & Diabetic nephropathy & $1.4 \pm 0.7$ & $0.8 \pm 1.4$ & 0.284 \\
\hline & Others & $1.0 \pm 1.3$ & $0.3 \pm 0.6$ & 0.048 \\
\hline \multirow[t]{2}{*}{ Time to evacuation } & Diabetic nephropathy & $1.1 \pm 0.8$ & $0.8 \pm 0.9$ & 0.149 \\
\hline & Others & $0.7 \pm 0.6$ & $0.5 \pm 0.7$ & 0.530 \\
\hline \multirow[t]{2}{*}{ Assisted bowel movements } & Diabetic nephropathy & $1.0 \pm 0.0$ & $1.0 \pm 0.0$ & $\dagger$ \\
\hline & Others & $1.3 \pm 0.5$ & $0.7 \pm 0.5$ & 0.020 \\
\hline \multirow[t]{2}{*}{ Number of bowel movements failed/24 h } & Diabetic nephropathy & $1.0 \pm 0.5$ & $0.4 \pm 0.5$ & 0.037 \\
\hline & Others & $1.2 \pm 0.4$ & $0.2 \pm 0.4$ & 0.003 \\
\hline \multirow[t]{2}{*}{ Duration (years) of suffering from disturbed defecation } & Diabetic nephropathy & $1.1 \pm 0.6$ & $1.1 \pm 0.6$ & - \\
\hline & Others & $1.1 \pm 1.0$ & $1.4 \pm 1.4$ & - \\
\hline
\end{tabular}

Results of analysis: diabetic nephropathy $n=8$, others $n=11$

Statistical analysis was performed using the paired $t$-test (spontaneous stool frequency) or Wilcoxon signed-rank test (CSS scores)

No statistical analysis was performed on the duration of suffering from disturbed defecation

tThe $p$ value could not be calculated

Elobixibat reduced the IDWG and IP significantly. IDWG is believed to be nearly equivalent to the amount of fluid intake [18]; therefore, the elobixibat-induced decrease in IDWG may have been caused by an increased water secretion into the colon lumen due to elobixibat. Moreover, the decreased IP that was observed in this study had also previously been found in lubiprostone, a laxative with a different mechanism of action [19], suggesting that constipation relief and reduction of phosphorus absorption by shortening the duration of stool retention, as well as an enhancement of the effect of phosphate binders, may have contributed to the above.

The side effects observed in our study consisted of diarrhea, abdominal pain, and nausea, and their respective incidence rates showed no considerable difference with the findings of a previous placebo-controlled, randomized double-blind study [14] conducted on patients with chronic constipation. Furthermore, abusive use of laxatives has previously been reported to be associated with decreased blood potassium concentrations [20]; however, in our study, elobixibat did not affect serum albumin levels or the blood concentration of electrolytes other than IP. A previous report has shown that elobixibat caused a mild decrease in LDL-C levels because cholesterol, a precursor of bile acids, is used for bile acid biosynthesis [7]. However, in our study, LDL-C levels showed a tendency to decrease, but there was no statistically significant difference.

The use of a phosphate binder is one of the causes of constipation in hemodialysis patients [2]. In our study, elobixibat increased the frequency of spontaneous bowel movement and reduced the total CSS score and all CSS subscores, except for the duration of disturbed defecation, both in patients with and without phosphate binder treatment. These results indicate that elobixibat was useful for the improvement of constipation symptoms, regardless of the use of a phosphate binder.

Patients with chronic constipation have been reported to have a reduced survival rate due to an elevated risk of death from cardiovascular disease $[4,5]$. Previous reports have shown that in maintenance hemodialysis patients, a high IDWG was associated with mortality [21]. Further, vascular calcification due to hyperphosphatemia has also been reported to increase the risk of cardiovascular death [22], and strict phosphorus control is believed to contribute to an improved vital prognosis [23]. In our 
Table 4 Comparison of spontaneous stool frequency and constipation score by the use of phosphate binder

\begin{tabular}{|c|c|c|c|c|}
\hline \multirow[t]{2}{*}{ Parameter } & \multirow{2}{*}{$\begin{array}{l}\text { Use of } \\
\text { phosphate } \\
\text { binder }\end{array}$} & \multicolumn{2}{|l|}{ Mean \pm SD } & \multirow{2}{*}{$\begin{array}{l}p \\
\text { value }\end{array}$} \\
\hline & & Baseline & Week 12 after administration & \\
\hline \multirow[t]{2}{*}{ Spontaneous stool frequency } & Yes & $1.8 \pm 0.6$ & $4.9 \pm 2.3$ & $<0.001$ \\
\hline & No & $2.6 \pm 0.9$ & $5.2 \pm 2.6$ & 0.033 \\
\hline \multirow[t]{2}{*}{ Total CSS score } & Yes & $10.1 \pm 3.1$ & $5.7 \pm 2.7$ & 0.002 \\
\hline & No & $11.2 \pm 3.5$ & $5.8 \pm 4.8$ & 0.058 \\
\hline \multirow[t]{2}{*}{ Stool frequency } & Yes & $0.8 \pm 0.6$ & $0.4 \pm 0.6$ & 0.041 \\
\hline & No & $1.4 \pm 0.9$ & $0.4 \pm 0.5$ & 0.089 \\
\hline \multirow[t]{2}{*}{ Dyschezia } & Yes & $1.8 \pm 1.0$ & $0.5 \pm 0.7$ & 0.002 \\
\hline & No & $2.2 \pm 0.8$ & $0.8 \pm 1.3$ & 0.134 \\
\hline \multirow[t]{2}{*}{ A sensation of incomplete evacuation } & Yes & $2.2 \pm 1.3$ & $1.3 \pm 1.4$ & 0.091 \\
\hline & No & $2.0 \pm 1.2$ & $1.4 \pm 1.5$ & 0.149 \\
\hline \multirow[t]{2}{*}{ Abdominal pain } & Yes & $1.3 \pm 1.1$ & $0.6 \pm 1.2$ & 0.101 \\
\hline & No & $0.8 \pm 0.8$ & $0.2 \pm 0.4$ & 0.149 \\
\hline \multirow[t]{2}{*}{ Time to evacuation } & Yes & $0.9 \pm 0.8$ & $0.6 \pm 0.6$ & 0.182 \\
\hline & No & $1.0 \pm 0.7$ & $0.8 \pm 1.1$ & 0.773 \\
\hline \multirow[t]{2}{*}{ Assisted bowel movements } & Yes & $1.1 \pm 0.4$ & $0.9 \pm 0.3$ & 0.149 \\
\hline & No & $1.2 \pm 0.4$ & $0.6 \pm 0.5$ & 0.149 \\
\hline \multirow[t]{2}{*}{ Number of bowel movements failed/24 $\mathrm{h}$} & Yes & $1.1 \pm 0.5$ & $0.3 \pm 0.5$ & 0.002 \\
\hline & No & $1.0 \pm 0.0$ & $0.2 \pm 0.4$ & 0.072 \\
\hline \multirow[t]{2}{*}{ Duration (years) of suffering from disturbed defecation } & Yes & $0.9 \pm 0.5$ & $1.2 \pm 1.0$ & - \\
\hline & No & $1.6 \pm 1.5$ & $1.4 \pm 1.7$ & - \\
\hline
\end{tabular}

Results of analysis: patients with phosphate binder treatment $n=14$, patients without phosphate binder treatment $n=5$

Statistical analysis was performed using the paired $t$-test (spontaneous stool frequency) or Wilcoxon signed-rank test (CSS scores)

No statistical analysis was performed on the duration of suffering from disturbed defecation

study, elobixibat showed not only an improving effect on constipation symptoms, but also a reducing effect on IP and IDWG, suggesting that elobixibat was useful for phosphorus control and IDWG control in hemodialysis patients; therefore, it may potentially lead to an improvement of the vital prognosis of hemodialysis patients.

Moreover, previous reports have shown that elobixibat takes approximately $4-5 \mathrm{~h}$ to trigger the first bowel movement and that although the time from medication to defecation differed from one individual to another, it was nearly constant for each individual [24]. Therefore, improvements in the timing of the ingestion of elobixibat may potentially help avoid the urge to defecate during hemodialysis and may contribute to an improvement of QOL in maintenance hemodialysis patients.

Our study shows the results of a retrospective analysis of medical records of maintenance hemodialysis patients in a single institution, but in the future, prospective multicenter studies will need to be conducted on hemodialysis patients, and the efficacy and safety and QOL-improving effect of a long-term administration of elobixibat will need to be examined.

\section{Conclusions}

Our findings showed that elobixibat was effective in improving constipation symptoms in maintenance hemodialysis patients with chronic constipation. Additionally, elobixibat improved the IDWG and IP levels, which are dialysis-related laboratory values. Our findings also suggested that elobixibat may be useful as a laxative in maintenance hemodialysis patients with chronic constipation.

\section{Abbreviations}

CTR: Clinical Trials Registry; QOL: Quality of life; BSFS: Bristol Stool Form Scale; IDWG: Interdialytic weight gain; IP: Inorganic phosphorus; Alb: Albumin;

CSS: Constipation score system; LDL-C: Low-density lipoprotein cholesterol

\section{Acknowledgement}

We thank all contributors to our study.

\section{Authors' contributions}

TS was responsible for planning, conducting, and reporting this work. TS and $\mathrm{HH}$ considered and agreed with manuscript results, discussion, and contributions. All authors read and approved the final manuscript.

\section{Funding}

Funding for this study was provided by Mochida Pharmaceutical Co., Ltd., and EA Pharma Co., Ltd. 


\section{Availability of data and materials}

The datasets analyzed in the present study are available from the corresponding author upon reasonable request.

\section{Competing interest}

TS has received funding from the following companies: Mochida Pharmaceutical Co., Ltd., and EA Pharma Co., Ltd. HH has no competing interests.

\section{Ethics approval and consent to participate}

This research protocol has the approval of the ethical review board of Yamauchi Clinic on July 2, 2019 (approval code 2019-06-00064). This protocol was also registered with the UMIN-CTR (UMIN000037285) on July 5, 2019. The study was conducted in compliance with the standard of the 1964 Helsinki Declaration, its later amendments, and Ethical Guidelines for Medical and Health Research Involving Human Subject or comparable ethical standards. Informed consent was obtained in the form of opt-out on the inhospital bulletin board for 3 months.

\section{Consent for publication}

Not applicable.

Received: 23 January 2020 Accepted: 30 March 2020

Published online: 21 April 2020

\section{References}

1. Masakane I, Taniguchi M, Nakai S, Tsuchida K, Wada A, Ogata S, et al. Annual Dialysis Data Report 2016, JSDT Renal Data Registry. Renal Replacement Therapy. 2018:4:45

2. Yasuda G, Shibata K, Takizawa T, Ikeda Y, Tokita Y, Umemura S, et al. Prevalence of constipation in continuous ambulatory peritoneal dialysis patients and comparison with hemodialysis patients. Am J Kidney Dis. 2002; 39(6):1292-9.

3. Zhang J, Huang C, Li Y, Chen J, Shen F, Yao Q, et al. Health-related quality of life in dialysis patients with constipation: a cross-sectional study. Patient Prefer Adherence. 2013:7:589-94.

4. Chang JY, Locke GR 3rd, McNally MA, Halder SL, Schleck CD, Zinsmeister AR. Impact of functional gastrointestinal disorders on survival in the community. Am J Gastroenterol. 2010;105(4):822-32

5. Honkura K, Tomata $Y$, Sugiyama K, Kaiho Y, Watanabe T, Zhang S, et al. Defecation frequency and cardiovascular disease mortality in Japan: The Ohsaki cohort study. Atherosclerosis. 2016;246:251-6.

6. Foley RN, Parfrey PS, Sarnak MJ. Epidemiology of cardiovascular disease in chronic renal disease. J Am Soc Nephrol. 1998;9:S16-23.

7. Guerrera MP, Volpe SL, Mao JJ. Therapeutic uses of magnesium. Am Fam Physician. 2009;80(2):157-62.

8. Locke GR 3rd, Pemberton JH, Phillips SF. AGA technical review on constipation. American Gastroenterological Association. Gastroenterology. 2000;119(6):1766-78.

9. Study Group related to the Japanese Society of Gastroenterology: Study Group for the Diagnosis and Treatment of Chronic Constipation. The 2017 Clinical Practice Guidelines for Chronic Constipation. Nankodo, Tokyo.

10. Acosta A, Camilleri M. Elobixibat and its potential role in chronic idiopathic constipation. Ther Adv Gastroenterol. 2014;7(4):167-75.

11. Taniguchi S, Yano T, Imaizumi M, Manabe N. Elobixibat, an ileal bile acid transporter inhibitor, induces giant migrating contractions during natural defecation in conscious dogs. Neurogastroenterol Motil. 2018;30(12):e13448.

12. Wingate DL, Krag E, Mekhjian HS, Phillips SF. Relationships between ion and water movement in the human jejunum, ileum and colon during perfusion with bile acids. Clin Sci Mol Med. 1973;45(5):593-606.

13. Bampton PA, Dinning PG, Kennedy ML, Phillips SF. The proximal colonic motor response to rectal mechanical and chemical stimulation. Am J Physiol Gastrointest Liver Physiol. 2002;282(3):G443-9.

14. Nakajima A, Seki M, Taniguchi S, Ohta A, Gillberg PG, Mattsson JP, et.al. Safety and efficacy of elobixibat for chronic constipation: results from a randomised, double-blind, placebo-controlled, phase 3 trial and an open-label, single-arm, phase 3 trial. Lancet Gastroenterol Hepatol 2018; 3(8):537-547.

15. Lacy BE, Mearin F, Chang L, Chey WD, Lembo AJ, Simren M, et al. Bowel disorder. Gastroenterology. 2016;150(6):1393-407.
16. Lewis SJ, Heaton KW. Stool form scale as a useful guide to intestinal transit time. Scand J Gastroenterol. 1997:32(9):920-4.

17. Agachan F, Chen T, Pfeifer J, Reissman P, Wexner SD. A constipation scoring system to simplify evaluation and management of constipated patients. Dis Colon Rectum. 1996;39(6):681-5.

18. López-Gómez JM, Villaverde M, Jofre R, Rodriguez-Benítez P, Pérez-García R. Interdialytic weight gain as a marker of blood pressure, nutrition, and survival in hemodialysis patients. Kidney Int Suppl. 2005;93:S63-8.

19. Gen S, Nobe K, Ikeda N. Lubiprostone, a novel laxative, might improve hyperphosphatemia without water dilution. Ren Replace Ther. 2016;2:50

20. Cummings JH. Laxative abuse. Gut. 1974;15(9):758-66.

21. Foley RN, Herzog CA, Collins AJ. United States Renal Data System. Blood pressure and long-term mortality in United States hemodialysis patients: USRDS Waves 3 and 4 Study. Kidney Int. 2002;62(5):1784-90.

22. Tentori F, Blayney MJ, Albert JM, Gillespie BW, Kerr PG, Bommer J, et al. Mortality risk for dialysis patients with different levels of serum calcium, phosphorus, and PTH: The Dialysis Outcome and Practice Patterns Study (DOPPS). Am J Kidney Dis. 2008:52:519-30.

23. Taniguchi M, Fukagawa M, Fujii N, Hamano T, Shoji T, Yokoyama K, et al. Serum phosphate and calcium should be primarily and consistently controlled in prevalent hemodialysis patients. Ther Apher Dial. 2013;17:221-8.

24. Odaka T. Clinical effectiveness of elobixibat by administration before dinner in patients with chronic constipation. Journal of the Japanese Society of Gastroenterology. in press.

\section{Publisher's Note}

Springer Nature remains neutral with regard to jurisdictional claims in published maps and institutional affiliations.

\section{Ready to submit your research? Choose BMC and benefit from:}

- fast, convenient online submission

- thorough peer review by experienced researchers in your field

- rapid publication on acceptance

- support for research data, including large and complex data types

- gold Open Access which fosters wider collaboration and increased citations

- maximum visibility for your research: over $100 \mathrm{M}$ website views per year

At $\mathrm{BMC}$, research is always in progress.

Learn more biomedcentral.com/submissions 